\title{
Effects of Silica Content on the Properties of Poly(N-isopropylacrylamide) / Silica Nanocomposite Hydrogels
}

\author{
Lili Wu ${ }^{1,2}$, Chao Zhang ${ }^{1}$ and Chaocan Zhang ${ }^{1}$ \\ ${ }^{1}$ School of Materials Science and Engineering, Wuhan University of Technology, Wuhan, Hubei, \\ 430070, P. R. China \\ ${ }^{2}$ School of Chemistry and Chemical Engineer, Shaanxi University of Science \&Technology, \\ Xi'an,Shanxi, 710021,P. R. China \\ polym_wl@whut.edu.cn
}

\begin{abstract}
Keywords: Poly(N-isopropylacrylamide); Silica sol; Composite hydrogels; Mechanical; Gelation; Thermosensitive.

Abstract: Poly(N-isopropylacrylamide) is a representative thermosensitive polymer, whose hydrogels have broad applications. But poly(N-isopropylacrylamide) hydrogels are weak and brittle. To get higher strength hydrogels, in this paper, silica sol was utilized as nanoparticle reinforcer source to construct poly(N-isopropylacrylamide)/silica nanocomposite hydrogels by in situ polymerization. By means of scanning electron microscopy, nano-silica was found dispersed evenly on the surface of PNIPA polymer network. Encouragingly, the compression strength of composite hydrogels have been enhanced more than $60 \%$ owing to the introduction of nano-silica. Obviously, the more silica sol this composite hydrogels contain, the longer gelation time they obtain. Compared to pure PNIPA hydrogels, nanocomposite hydrogels have faster deswelling speed at high temperature. And the lower critical solution temperature of nanocomposite hydrogels with different content of silica hydrogels was found between $33.5^{\circ} \mathrm{C}$ to $34.5^{\circ} \mathrm{C}$. Because silica sol is facial to composite with hydrogel, this study provides a simple way for nanocomposite reinforced hydrogels preparation.
\end{abstract}

\section{Introduction}

Hydrogels are commonly defined as being cross-linked polymer network with high capacity for absorbing either water or biological fluids. Due to its particular feedback system [1], that hydrogel can show significant changes in shapes and volumes as a response to variation of external stimuli such as temperature [2], $\mathrm{pH}$ [3] and electric field [4], hydrogels have gained its popular application in numerous fields such as biomaterials [5] and sensing applications [6]. Poly(N-isopropylacrylamide)( PNIPA) is a representative thermosensitive polymer with a lower critical solution temperature(LCST) around $34{ }^{\circ} \mathrm{C}$ [7], a specific temperature at which the polymer shows phase separation. Due to this, PNIPA hydrogel have won great interests for study on various applications such as drug delivery systems [8] and pharmaceutical application [9]. However, most traditional hydrogels are weak and brittle. In the past decades, efforts have been made to improve mechanical properties of these hydrogels. Some nano-particles, such as clay [10], adamantly methacrylate [11] and calcium alginate [12], have been used as reinforcers to enhance the mechanical properties of PNIPA hydrogels. In the previous researches, PNIPA/clay nanocomposite hydrogels exhibited excellent mechanical properties. Xiang et al. [13] prepared A new polymer/clay nano-composite hydrogel, it has significantly improved tensile mechanical properties. Liu [14] reported high clay content nanocomposite hydrogels with surprising mechanical strength, of which tensile strength can reach $1 \mathrm{MPa}$, and elongation at break can run up to $1400 \%$.

Silica sol is a kind of colloid which is formed by $\mathrm{SiO} 2$ particles with amorphous nano-scale pattern in water. Since the colloid particles are of nanometer $(10-20 \mathrm{~nm})$ and dispersed in water with low viscosity, it presents good dispersion and permeability when being mixed with other waterbase substances such as water solubility polymers. Because of the presence of hydroxyl group, silica particles could form hydrogen bonds to construct a space stereo 3D mesh structure in the system [15]. From this perspective, silica sol could be used as reinforcer nanoparticles the same as clay, and its 
dispersion in hydrogels would be more homogeneous than that of clay. But somehow, there are few reports about the introduction of silica sol to modify the properties of PNIPA hydrogels.

Although silica sol has not been introduced in PNIPA hydrogels before, it has gained its success for use in others nanocomposite hydrogels. Creton et al. [16] synthesized Poly(dimethylacrylamide)/silica hybrid hydrogels, showing that the absorption of silica nanoparticles on PDMA chains resulted in the formation of physical network and leaded to the increase in the compression strength and fracture toughness. Our previous research [17] synthesized poly(acrylamide)(PMA)/silica composite hydrogels, their compression strength and elastic modulus were significantly improved by adding silica sol compared with pure PAM hydrogels.

According to this idea, we focus on synthesizing a series of PNIPA/silica composite hydrogels by adding silica sol in N-isopropylacrylamide (NIPA) monomer and carrying out in situ free-radical polymerization in solution. We aim to investigate the influence of the amount of silica sol on the properties of hydrogels. Comparing with clay, silica sol is more facial to composite with hydrogel, so this paper provides a possibly simple way for nanocomposite reinforced hydrogels preparation.

\section{Experimental Methods}

\section{Materials.}

Silica sol, Jiangyin Chemical Co., Ltd (CH83-120, 20.24 wt\% SiO2, particles size: 4-5 nm, pH 8.5-9.0, the density is $1.14 \mathrm{~g} / \mathrm{cm} 3$ ). N-Isopropylacrylamide(NIPA; 98\%, Aladdin Chemistry Co.,Ltd). N, N'-Methylenebiscrylamide(BIS, 98\%, Sinopharm Chemistry Reagent Co.,Ltd). Ammonium persulfate(APS, 98\%, Sinopharm Chemistry Reagent Co.,Ltd). N, N, N, $\square$ N',-Tetramethylethylenediamine(TEMED, Sinopharm Chemistry Reagent Co.,Ltd).

\section{Preparation of PNIPA/Silica composite hydrogels.}

Formulations were chosen using the methodology proposes by as a reference [18] and were summarized in Table 1. Si0, Si5.0, Si7.5, Si10, and Si15 were used to represent the presence of different concentrations of Silica: 0, 5, 7.5, 10 and 15\% (Silica wt-\% based on the amount of NIPA).

Formulations were synthesized in a $50-\mathrm{mL}$ glass reactor under an $\mathrm{N} 2(\mathrm{~g})$ inert atmosphere. First, 5 $\mathrm{mL}$ of distilled water was added, and then followed by the monomer (NIPA), silica sol, the cross linking agent (BIS) and the initiator (APS). Finally, the accelerator (TEMED) were added successively at an inert atmosphere to continue the reaction at room temperature for $24 \mathrm{~h}$. To remove residual monomer NIPA and any other unreacted chemicals, the sample was immersed in distilled water for 24 $\mathrm{h}$ and the distilled water was refreshed every $8 \mathrm{~h}$. At last, the obtained hydrogel needed to be dried for $24 \mathrm{~h}$ under vacuum at $50{ }^{\circ} \mathrm{C}$ until that its weight didn't change any longer.

Table 1 Formulations prepared for synthesis of PNIPA+Silica hydrogels

\begin{tabular}{llllll}
\hline Material & Si0 & Si5.0 & Si7.5 & Si10 & Si15 \\
\hline Distilled water $(\mathrm{mL})$ & 5 & 5 & 5 & 5 & 5 \\
NIPA $(\mathrm{mg})$ & 400 & 400 & 400 & 400 & 400 \\
BIS $(\mathrm{mg})$ & 12 & 12 & 12 & 12 & 12 \\
APS $(\mathrm{mg})$ & 8 & 8 & 8 & 8 & 8 \\
TEMED $(\mu \mathrm{L})$ & 35 & 35 & 35 & 35 & 35 \\
Silica sol $(\mu \mathrm{L})$ & 0 & 90 & 135 & 180 & 270
\end{tabular}

NIPA: N-Isopropylacrylamide; BIS: N, N-Methylenebiscrylamide; APS: Ammonium persulfate; TEMED: N, N, N,'

$\mathrm{N}$, '-Tetramethylethylenediamine

\section{Characterization of PNIPA/ Silica composite hydrogels}

Infrared spectroscopy (FTIR). The prepared samples were analyzed with Fourier Transformed Infrared Spectroscopy(FTIR) using a Nicolet-170SXB spectrometer.

SEM morphology investigation. The prepared samples were observed by a Hitachi S-4800 field emission-scanning electron microscope (FE-SEM), at an acceleration voltage of $10.0 \mathrm{kV}$ [17]. 
Gelation behavior and LCST measurement by rheometer. Rheological measurements were carried out using a rheometer (TA instruments Rheology Division, AR 2000) with 40mm parallel plate geometry in oscillation shear mode. The mixture solution was put at the desired temperature $\left(10{ }^{\circ} \mathrm{C}\right)$ directly in the rheometer and then covered with mineral oil to prevent evaporation during the measurement [17]. In addition, storage modulus was supposed to record continously at the strain of $5 \%$ with the frequency of $1.0 \mathrm{rad} / \mathrm{s}$.

The thermal behavior or LCST behavior of the hydrogel sample was also determined by using the rheometer(TA instruments Rheology Division, AR 2000). After the hydrogel gelled, the thermal-mechanical analysis was performed from 20 to $45{ }^{\circ} \mathrm{C}$ at a heating rate of $0.5{ }^{\circ} \mathrm{C} / \mathrm{min}$, using $40 \mathrm{~mm}$ parallel plate geometry in oscillation shear mode.

Eequilibrium swelling ratio in different temperature. Each dried sample was swollen in an appropriate aqueous medium until equilibrium had been attained. The samples were held in distilled water for 24 hours to attain swelling equilibrium over a temperature range from 20 to $50{ }^{\circ} \mathrm{C}$. Each sample was then took out of the water bath and dabbed with filter paper to remove water on the surface. The swelling ratio was used to evaluate the swelling properties.

$\mathrm{SR}=\left(\mathrm{W}_{\mathrm{h}}-\mathrm{W}_{\mathrm{o}}\right) / \mathrm{W}_{\mathrm{o}}$

$\mathrm{W}_{\mathrm{h}}$ - the weight of the swollen sample, $\mathrm{W}_{\mathrm{o}}$ - the weight of the dry sample.

Mechanical Properties Measurement. The compression tests were performed on hydrogels with 2 $\mathrm{mm} / \mathrm{min}$ of the loading velocity. In addition, the compression test required hydrogel samples shaped in the cylindrical molds $(\square 22 \mathrm{~mm} \times 17 \mathrm{~mm})$.

Deswelling Kinetics Measurement. First, the dry sample was immersed in the distilled water at $20^{\circ} \mathrm{C}$ for 24 hours so that it changed into a fully swollen one. Then this gel block was transferred into the distilled water at $50{ }^{\circ} \mathrm{C}$ and accurate measurement of the swollen weight at regular time intervals was necessary. After wiping off the water on the surface with wet filter paper, the weight of the hydrogel sample was recorded. The water retention was defined as follows:

$\mathrm{SR}=\left(\mathrm{W}_{\mathrm{t}}-\mathrm{W}_{\mathrm{o}}\right) / \mathrm{W}_{\mathrm{o}}$

$\mathrm{W}_{\mathrm{t}}$ - the weight of the swollen sample at time $\mathrm{t}, \mathrm{W}_{\mathrm{o}}$ - the weight of the dry sample.

\section{Results and discussion}

\section{FTIR Spectroscopy}

Fig 1 shows the FTIR spectra comparison of PNIPA and PNIPA/silica hydrogels. It can be seen from the figure that the two curves basically have the same trend. Among these, the band at $3441 \mathrm{~cm}^{-1}$ and $3315 \mathrm{~cm}^{-1}$ are due to the N-H stretching of PNIPA, and the appearance of peak at $3072 \mathrm{~cm}^{-1}$ is attributed to the associated N-H of PNIPA. The characteristic bands of polymer main chain for C-H (-CH3, - CH2-) are shifted to 2800-3000 $\mathrm{cm}^{-1}$. The peaks at $1648 \mathrm{~cm}^{-1}$ and $1538 \mathrm{~cm}^{-1}$ are assigned to the $\mathrm{C}=\mathrm{O}$ in amide area $\mathrm{I}$ and the $\mathrm{N}-\mathrm{H}$ and the $\mathrm{C}-\mathrm{N}$ in amide area II respectively. The band from $1300-1500 \mathrm{~cm}^{-1}$ are due to the stretching of $\mathrm{CH} 3(-\mathrm{CH}(\mathrm{CH} 3) 2)$. The band at $1171 \mathrm{~cm}^{-1}$ is attributed to the C-N stretching in amide area III. The peaks in Fig 1(a) at $1109 \mathrm{~cm}^{-1}$ is assigned to the Si-O, which clearly identified the presence of $\mathrm{SiO} 2$ in hydrogels.

\section{Structure of Composite Hydrogels}

After the samples were dried to constant weight under vacuum, scanning electron microscopy (SEM) was used to study the distribution of silica particles and the porous structure of the pure gels and hybrid gels. In Figure 2(Left), the pure PNIPA hydrogel gel has smooth surface. As being shown in Figure 2(Right), silica particles appear in nano degree and distribute homogeneously without forming clusters and aggregation phenomenon, which suggests that the silica particles can composite in PNIPA at nanometer level by simply mixing silica sol in the NIPA water solution, and then polymerizing in situ. 


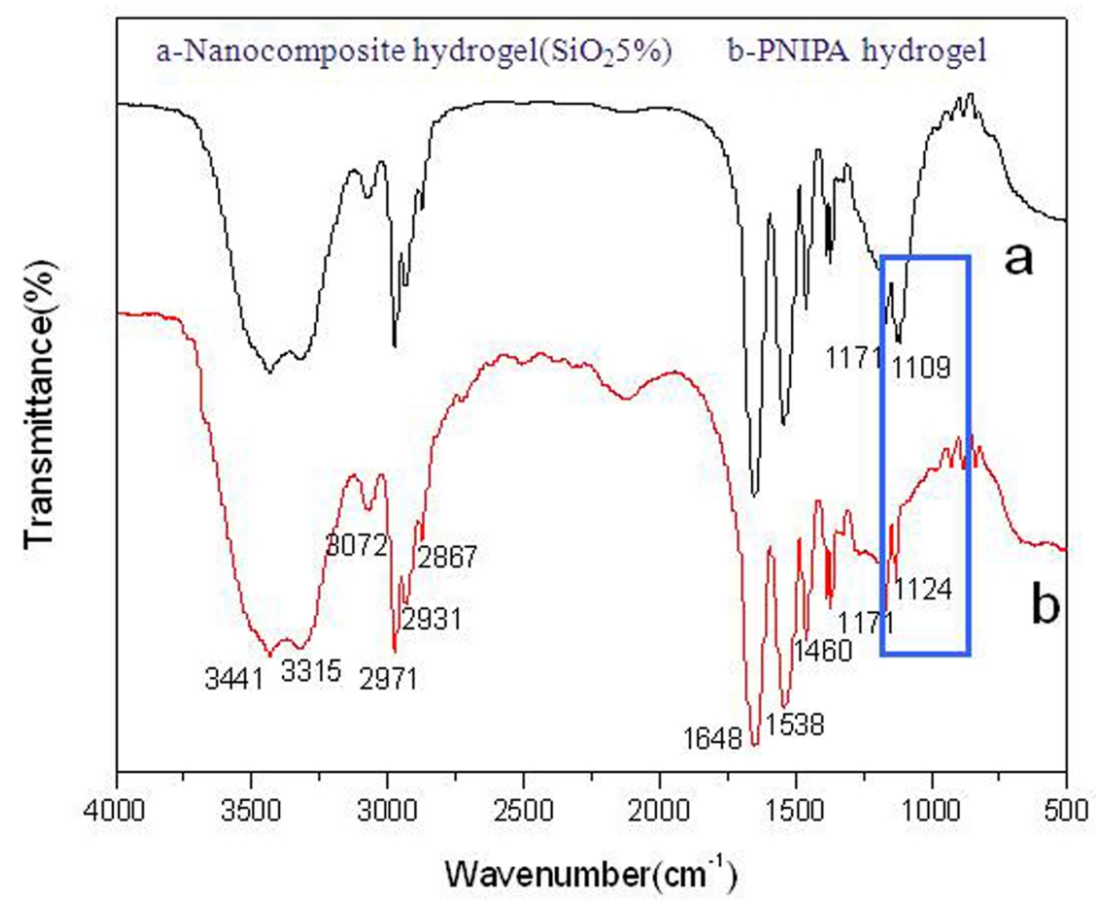

Fig. 1 FTIR spectra of (a) dried PNIPA/silica nanocomposite gel (Si5.0), (b) dried PNIPA gel (Si0)
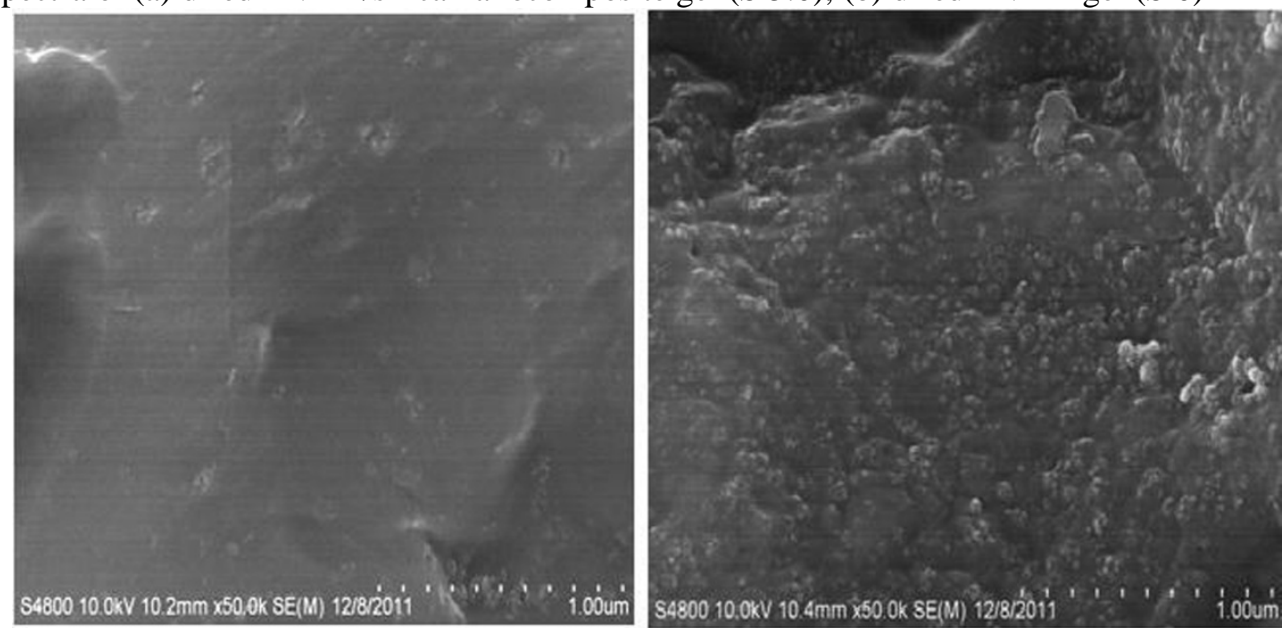

Fig. 2 SEM images of the fracture surface of dry PNIPA/silica sol composite gels Si0(Left), Si15(Right)(drying at 50 ${ }^{\circ} \mathrm{C}$ in vacuum)

\section{Gelation process of Composite Hydrogels}

Figure 3 shows the storage modulus G' versus time, which is used to describe the gelling kinetics of PNIPA/silica composite hydrogels. Changes of G' from 3 minutes to 120 minutes is observed in order to investigate the influence of introduction of silica sol on gelation process of hydrogels. At a certain time point to, G' suddenly rises as a result of the cross-linking network structure forming. Gelation process always lasts for some time and to is referred as the critical sol-gel transition point which can be shown on the time course of storage modulus G' [19]. As being shown in figure 3 the gel forming time of PNIPA/silica composite hydrogels gradually increases along with the increasing content of silica. The G' curves show that it needs time to cross-link, the longer gel forming time suggests that the increasing amount of silica hindered the gelation process. It is confirmed that the composite hydrogels have been proposed to form both a high degree of heterogeneity in their network, due to the delocalized fracture of the bonds of the network in a large volume [16]. So it could be conjectured that the introduction of silica sol has an influence on the dissipation and chain relaxation due to the interactions between the polymer chains and silica nanoparticles. 


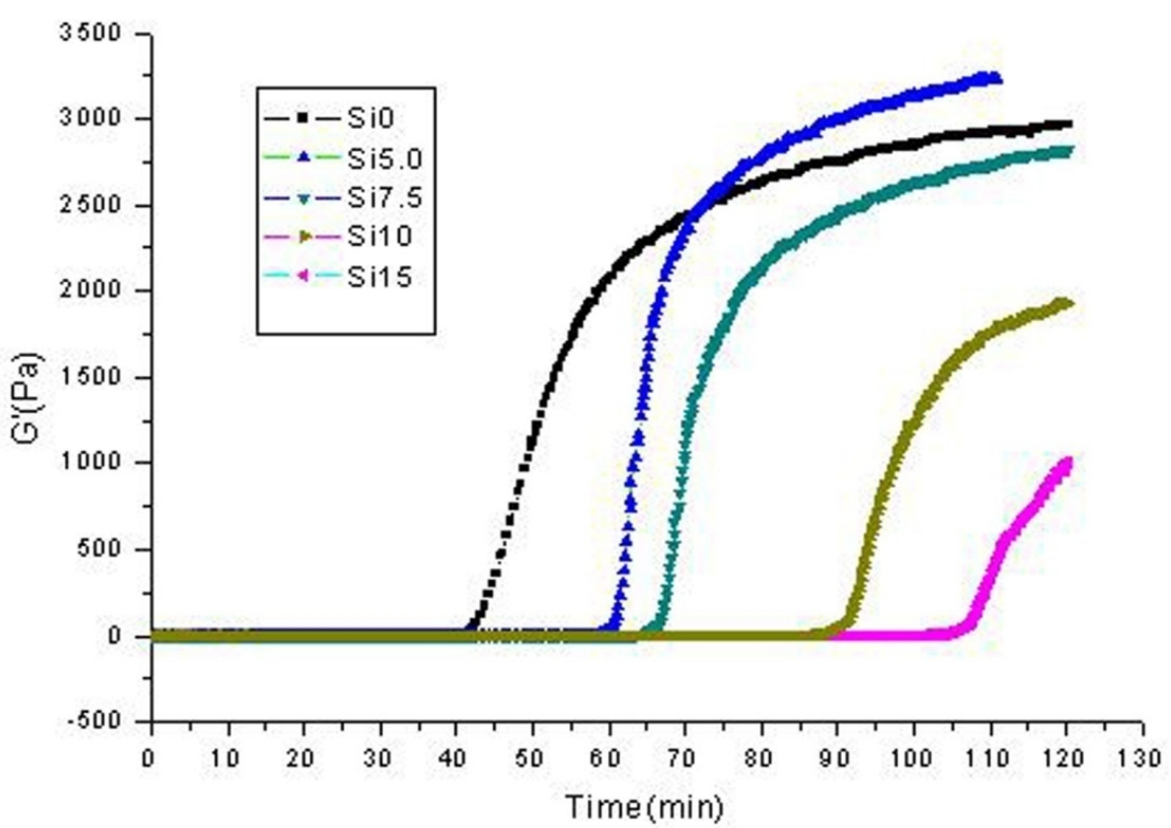

Fig. 3 The storage modulus G' of composite hydrogels as a function of polymerization time for different silica content Table 2 LCSTs of the Swollen Composite PNIPA Hydrogels

\begin{tabular}{lccccc} 
Sample & Si0 & Si5.0 & Si7.5 & Si10 & Si15 \\
\hline Silica $\operatorname{sol}(\mu \mathrm{L})$ & 0 & 90 & 135 & 180 & 270 \\
$\operatorname{LCST}\left({ }^{\circ} \mathrm{C}\right)$ & 34.5 & 34.4 & 34.0 & 33.5 & 34.1
\end{tabular}

LCST: lower critical solution temperature

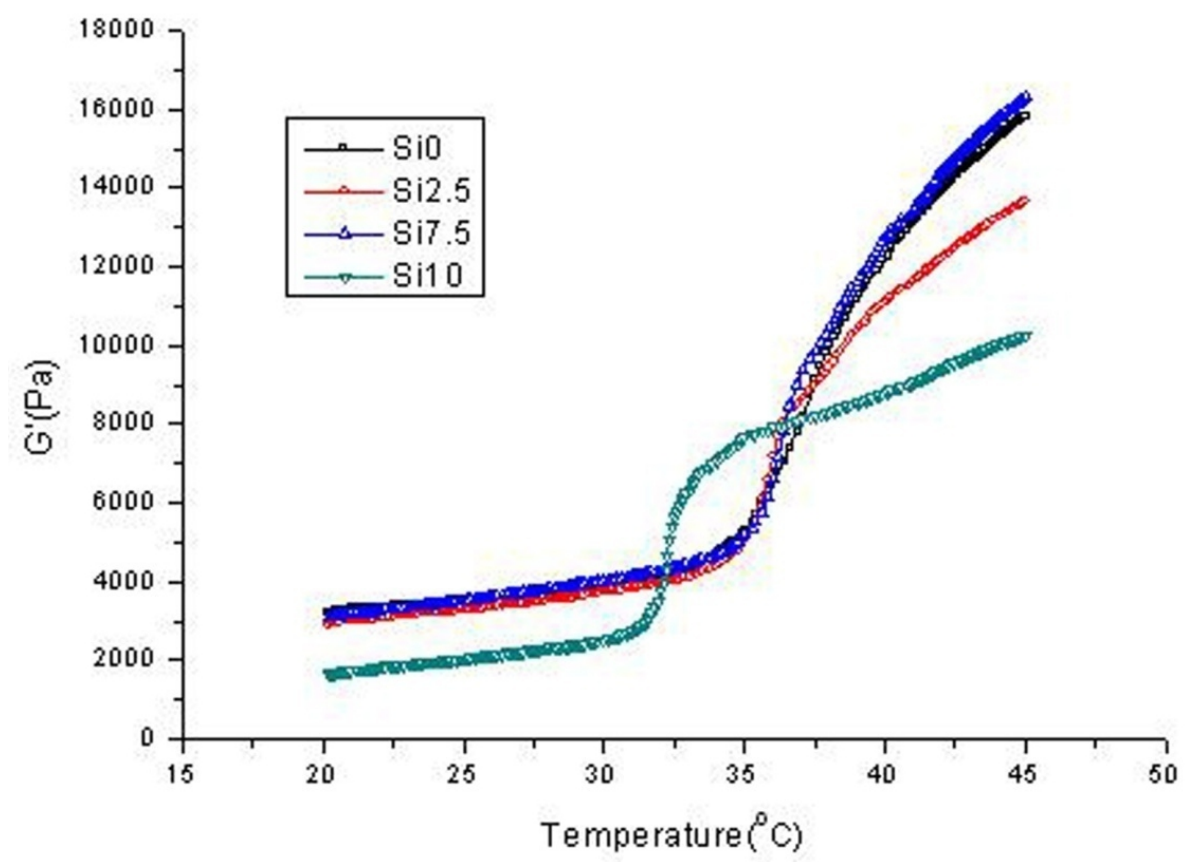

Fig. 4 Rheological behavior (G') for synthesized PNIPA and PNIPA/silica composite hydrogels in function of temperature

\section{LCST of Composite Hydrogels}

Few research works [20-22] used a dynamic temperature rheological ramp to measure the LCST phase transition for PNIPA hydrogels. The dynamic thermal analysis of the ordinary and modified PNIPA hydrogels is shown in Figure 4. The data (Table 2) indicate that the composite PNIPA hydrogels have similar LCSTs which around $34{ }^{\circ} \mathrm{C}$; In other words, the introduction of silica sol into the PNIPA 
hygrogels has no obvious impact on LCST. Furthermore, the amount of silica sol also has no much influence on the LCST. As was shown in Figure 4, a significant change in G' indicates the phase transition. The LCST is determined by the intersecting point of two tangent lines from the baseline and slope of the endothermic peak, which is as reported before around $34{ }^{\circ} \mathrm{C}$ [7].

As well-known, the phase transition of a thermosensitive polymer is a result of the change in its hydrophilic-hydrophobic balance upon external temperature variations [23]. NIPA chains are hydrophilic, soluble and surrounded by water as individual random-coil chains when the temperature is lower than LCST. That is, water acts as the lubricant among the chains, making molecular mobilization easier and thus reducing the value of G' [22]. When the temperature increases, the polymer chains become hydrophobic and insoluble in water because of the thermal dissociation of hydrating water molecules from the polymer chains. Losing water means that it is harder to mobilize the chains, which show an increase in the elastic component of the modulus G'.

According to the Table 2, the pure PNIPA hydrogels have a LCST higher a little than the LCST of PNIPA/silica composite hydrogels. It indicates that the introduction of silica sol is helpful to lubricate the polymer chain so that PNIPA molecules will be easier to move and phase transition needs less energy, which result in the decrease of LCST of PNIPA/silica composite hydrogels.

\section{Equilibrium swelling ratio}

In this section, the temperature dependence of swelling ratio of the composite hydrogels was studied to determine the temperature-sensitive properties. Figure 5 shows the temperature dependence of swelling ratios over a temperature range from 20 to $50{ }^{\circ} \mathrm{C}$. From the Figure 5, all the composite hydrogels demonstrate a similar trend, that is, the swelling ratios of all the gels decrease rapidly as temperature increases. Specifically, the highest swelling ratios of PNIPA/Silica sol are around 13, whereas the normal hydrogel has the swelling ratio more than 17 . Among modified hydrogels, with the increase of silica sol added, the swelling ratios decrease successively from 15 (Si5.0) to 11 (Silica15). The decreasing swelling ratios at room temperature may be explained as followings: due to the nanostructure of Silica sol, plenty of $\mathrm{SiO} 2$ take place of the holes of the network structure of PNIPA/Silica hydrogels, which hampers the entrance of water. secondly, the nanostructure of $\mathrm{SiO} 2$ may become the nucleus of the composite hydrogels. The more $\mathrm{SiO} 2$ become the nucleus, the more the density of the hydrogels increase around the nucleus, which leads to the decrease of swelling ratios. Plots in Figure also show that both the normal and modified hydrogels exhibit a similar temperature-dependent swelling ratio, but the thermo-induced swelling ratio changes of PNIPA/Silica hydrogels are smaller than the normal PNIPA. Upon heating, swelling ratios of all the hydrogels decrease steadily. As the temperature increases toward the phase transition point, swelling ratios decrease dramatically and the phase transition occurs. It is clear that LCSTs of all hydrogels were around $35^{\circ} \mathrm{C}$.

\section{Mechanical properties}

The Figure 6 shows the influence of introduction of silica sol on the mechanical behaviors of composite hydrogels. It is clear that the introduction of silica sol is beneficial especially to hybrid hydrogels when the amount of silica is $5 \mathrm{wt}-\%$, which exhibit excellent mechanical properties that the compression force increases by 63\%. However, the compression force drops slowly while the content of silica reaches to $7.5 \mathrm{wt}-\%$. After that, the tendency of compression force is continuous decreasing. The tendency of compression force variation may be explained as followings: at first, the increase of compression force was due to the silica nanoparticals, as the inorganic clays [13], act as multifunctional cross-linking agents through noncovalent interactions. With the increasing of silica content, the chemical cross-linking of polymer chain may be obviously hampered by the silica sol presence and it is proved indirectly from Fig 3. When the effect of chemical cross-linking hampered is more than the effect of the noncovalent interactions cross-linking between polymer chains and silica nanoparticals, the mechanical strength of the composite hydrogels will decrease. 


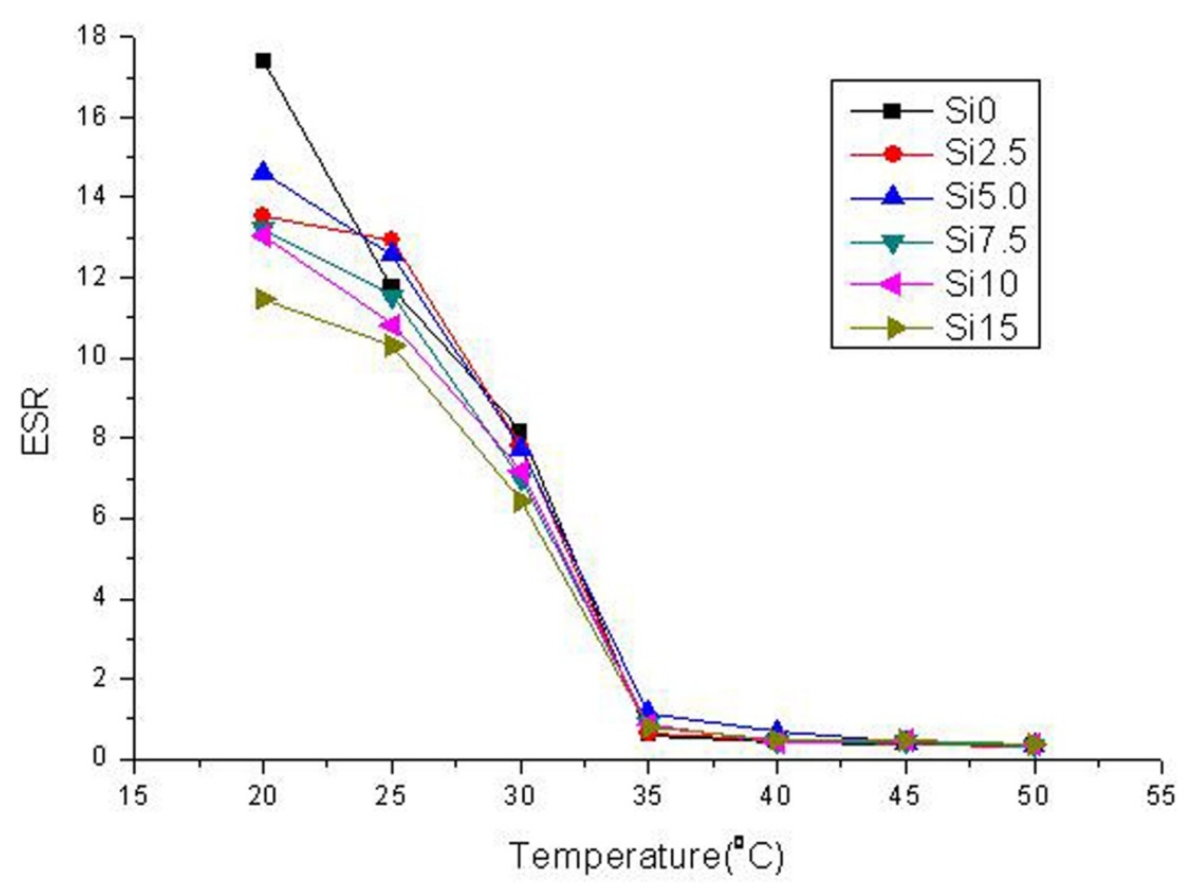

Fig. 5 Temperature dependence of swelling ratios of PNIPA/silica hydrogels

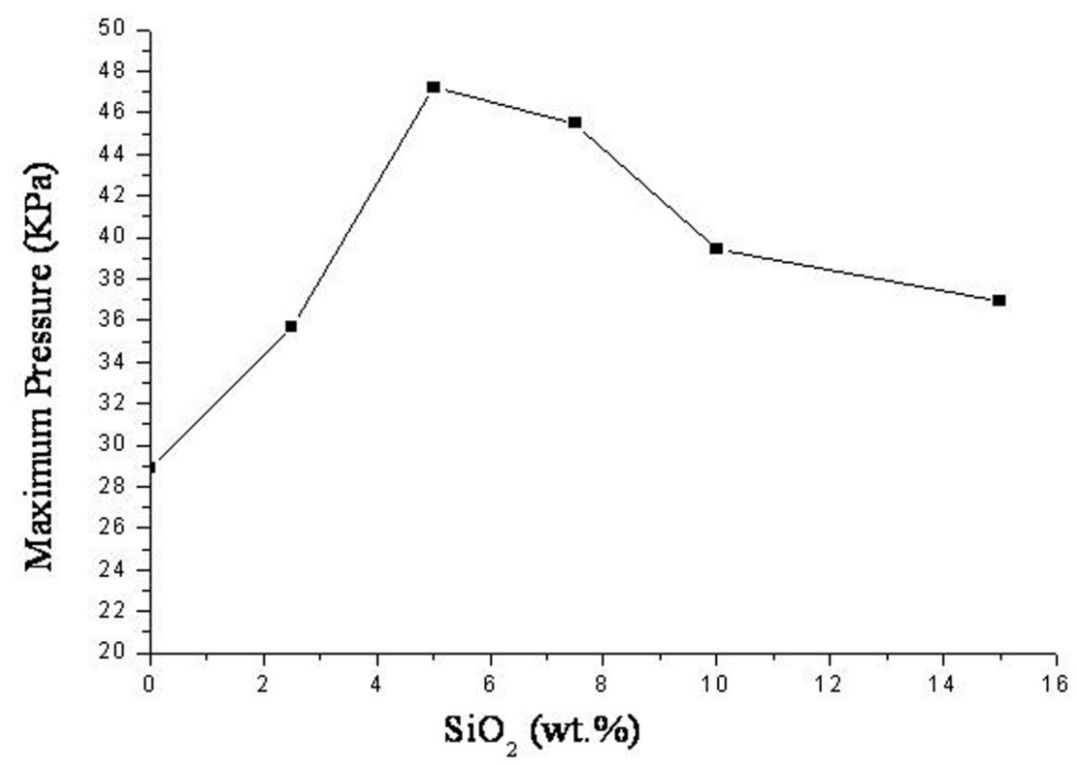

Fig. 6 Effects of silica sol content on compression properties of PNIPA/silica hydrogels

\section{Desweling kinetics}

From the Figure 7, it is shown that all the hydrogels tend to shrink and lost water once immersed into water at 60 oC. However, PNIPA/Silica hydeogels exhibit the faster shrinking rates than the normal PNIPA hydrogels. And with the amount of silica sol content increasing, the shrinking rate gets improved. Specially, the Si5.0 exhibits the greatest change of swelling rate. For example, the swelling ratio of Si0 decreases by $14 \%$ in half an hour, while that of Si5.0 decreases by $27 \%$ within the same time period. Because of the heterogeneous microstructure, many shrunk regions appear but with different collapsed phase during the shrinking process. As time goes by, free water in the water-rich regions may transfer into the surrounding dilute regions. Therefore, the water-rich regions connect with each other and an interconnected water release channel is generated throughout the hydrogel. Finally, the free water is evacuated quickly from the collapsing network, which results in the fast response rate of the heterogeneous hydrogel. Attributed to above reasons, the modified PNIPA hydrogels obtain the fast deswelling or shrinking rates upon the temperature jump from below LCST to above LCST [23]. 


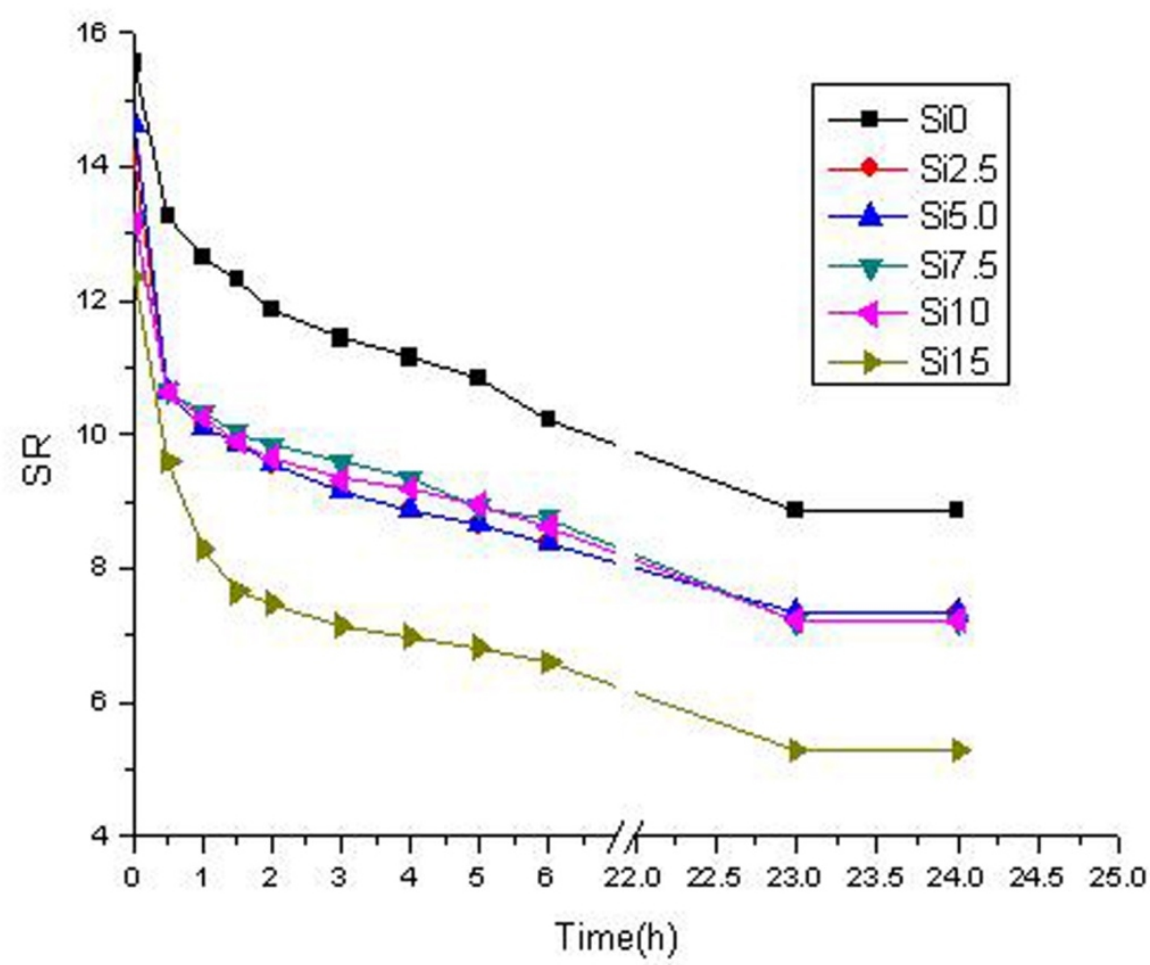

Fig. 7 Effects of silica sol content on deswelling kinetics of PNIPA/silica hydrogels at $60{ }^{\circ} \mathrm{C}$

\section{Conclusions}

Silica sol acting as the inorganic nanoparticle reinforcer has successfully brought about PNIPA/silica nanocomposite hydrogels. The smart nature is based on the LCST which is around $33.5-34.5{ }^{\circ} \mathrm{C}$. The modified composite hydrogels exhibit a stable response upon temperature changes. The investigation on the gelation reaction of PNIPA/silica nanocomposite hydrogels demonstrate the fact that increasing silica content hinders the PNIPA gel forming. In the PNIPA/silica nanocomposite hydrogels, the silica particles are uniformly distributed onto PNIPA network. Exactly as expected, silica particles make a difference in improvement of hybrids' mechanical properties. For example, the composite hydrogels acquire higher compress strength that maybe broaden their applications. All results indicate that the hybrids from formulation including NIPA and $5 \mathrm{wt}-\%$ silica sol acquire greatest potential to be used as a kind of stable and high-mechanical-performance hydrogels.

\section{Acknowledgements}

This study was funded by the State Scholarship Fund(201306955022).

\section{References}

[1] Okano T, Yamada N, Okuhara M, Sakai H. and Sakurai Y. Biomaterials 16(4):297-303 (1995)

[2] Romeo G and Fernandez-Nieves A. Adv Mater 22:3441-3445 (2010)

[3] Zarzar LD, Kim P, Aizenberg J. Adv Mate 23:1442-1446 (2011)

[4] Luo R, Li H, Birgersson E, Lam KY. J Biomedical Mater Res Part A 85(1):248-257 (2007)

[5] Paulino AT and Guilherme MR. Macromol Chem Phys 211:1196-1205 (2010)

[6] Hirose Y, Amiya T, Hirokawa Y and Tanaka T (1987) 20:1342-1344

[7] Taylor LD and Cerankowski LD. J Polym Sci, Polym Chem Edi 13:2551-2570 (1975)

[8] Qiu Y and Park K. Adv Drug Deliv Rev 53:321-339 (2001) 
[9] Groot CD, Luyn MV and Dijk-Wolthuis WV. Biomaterials22:1197-1203 (2001)

[10]Abdurrahmanoglu S and Okay O. J Appl Polym Sci 116:2328-2335 (2010)

[11]Zhu D, Lu M, Guo J, Liang L and Lan Y. J Appl Polym Sci 124:155-163 (2012)

[12]Petrusic S, Lewandowski M and Giraud S. J Appl Polym Sci124:890-903 (2012)

[13]Xiang Y, Peng Z and Chen D. Eur Polym J 42:2125-2132 (2006)

[14]Liu Y, Zhu M, Liu X, Zhang W, Sun B, Chen Y and Adler HP. Polymer 47:1-5 (2006)

[15]Labarrea D, Laurentb A and Lautiera A. Biomaterials 23:2319-2327 (2002)

[16]Lin W, Fan W, Marcellan A, Hourdet D and Creton C. 43:2554-2563 (2010)

[17]Wu L, Zeng L, Chen H and Zhang C. Polym Bull 68:309-316 (2012)

[18]Stile RA, Burghardt WR and Healy KE. Macromolecules 32:7370-7379 (1999)

[19]Ghosh P, Dev D and Samanta AK. J Appl Polym Sci 58:1727-1734 (1995)

[20] Ohya S, Sonoda H, Nakayama Y and Matsuda T. Biomaterials 26:655-659 (2005)

[21]Portehault D and Petit L. Coll Surf A 278:26-32 (2006)

[22] Coronado R, Pekerar S, Lorenzo AT and Sabino MA. Polym Bull 67:101-124 (2011)

[23]Xu X, Wang B and Wang Z. J Biomedical Mater Res 86:1023-1032 (2007) 